\title{
Research on Multimode Biometric Features Recognition System Adopting Neural Network
}

\author{
Wang Xiaosong ${ }^{1, *}$ and Zheng Zhiqing ${ }^{2}$ \\ ${ }^{I}$ Department of Management Science and Engineering, Shandong Institute of Business and Technology, Yantai, China; \\ ${ }^{2}$ Information Network Center, Shandong Institute of Business and Technology, Yantai, China
}

\begin{abstract}
The small sample issue is a common problem in face recognition system, and multi-modal model has strong generalization ability to solve the problem of small sample, which has already become the most important area of research in pattern recognition, however, the low accuracy and efficiency of the model has become a major challenge. Based on this, this paper proposes a efficient multimode biometric face and fingerprint recognition system based on neural network, which provides more efficient identification though choosing a good feature extraction and recognition algorithms. The Adoption of biometric recognition to authenticate a person's identity has greatly improved operational efficiency and the recognition accuracy in comparison with adoption of password or passphrase. The feasibility and effectiveness of the method in this paper has been verified in multimode biometric system database.
\end{abstract}

Keywords: Feature extraction, multi-level sensor, neural network, palm print recognition.

\section{INTRODUCTION}

Multimode biological feature recognition [1] is a pattern recognition research field to recognize identity based on a physical model of human or behavior patterns, and the advantages of biological feature is that it does not change or disappear. Many body parts, personal characteristics and imaging methods have been used to biological feature recognition system, such as fingers, hands, feet, eyes, ears, teeth, intravenous voice, signature, writing style, step-type and other features [2,3]. Each biological feature has its own advantages and limitations, so each biological feature is used for biological feature identification (authentication) application software. Adoption of biological feature recognition system in single feature has many limitations, such as there is noise upon censoring, without universality, between-class similarity, diversity within the class, deceptive and particularity, this single-mode biological feature recognition system may result in high false acceptance rate (FAR) and false rejection rate (FRR) [3, 4]. Biological feature recognition systems are fixed probabilistic, whose performances require adopting of basic background and key features to be evaluated. Biological feature recognition system adopts approximate matching tolerance, and observes biometric and biological properties and performance behavior of internal of people and between people [5-7]. Therefore, compared with the majority of the use of large binary result of information technology systems, biological feature recognition system provides probabilistic results. The new multi-mode face and fingerprint biological feature recognition system has better performance and is more popular than the non- multi-mode recognition system.
Multi-level perception (MLP) [8] is a forward artificial neural network model mapping input DS to the appropriate output DS, which is composed of a multi-layer nodes of the graph, with each layer connected to the next layer. In addition to the input node, each node is a processing unit with non-linear activation function. MLP application is called to train network back-propagation supervised learning techniques and characteristics in relation with ability of expression of forward MLP has also been authenticated [9]. As for Arbitrary function study, in the three layer networks, it is able to learn any function by adoption of any precision. As for continuous function study, in the two layer networks (the number of hidden units of function is evaluated based on the function), and it is able to learn every continuous function with limitation in a small error. As for Boolean function learning: in two layer network, each Boolean function can be learned, although the number of hidden processing units increases with input dimension index. It has one input unit, one layer of hidden units and the output unit is the two-layer network; the network has two layers of hidden units is threelayer network, and so on. The reason for this definition is that the input layer unit only acts only as an input channel, which has not been calculated as the number of layers.

\section{BIOLOGICAL FEATURE RECOGNITION SYSTEM}

Biological feature recognition system is an automatic identification based on their individual behavior and biological features. It is a individual tool used to deal with those that have been recognized (or not recognized), and thus to determine their belongings of group with permissions (or belonging of a group in rejected permission). This recognition depends on the assumption that an individual acts differently in physical and performance in different ways.

Figs. (1-3) illustrates the basic operation of the recognition process. Two basic operations in a common biological 


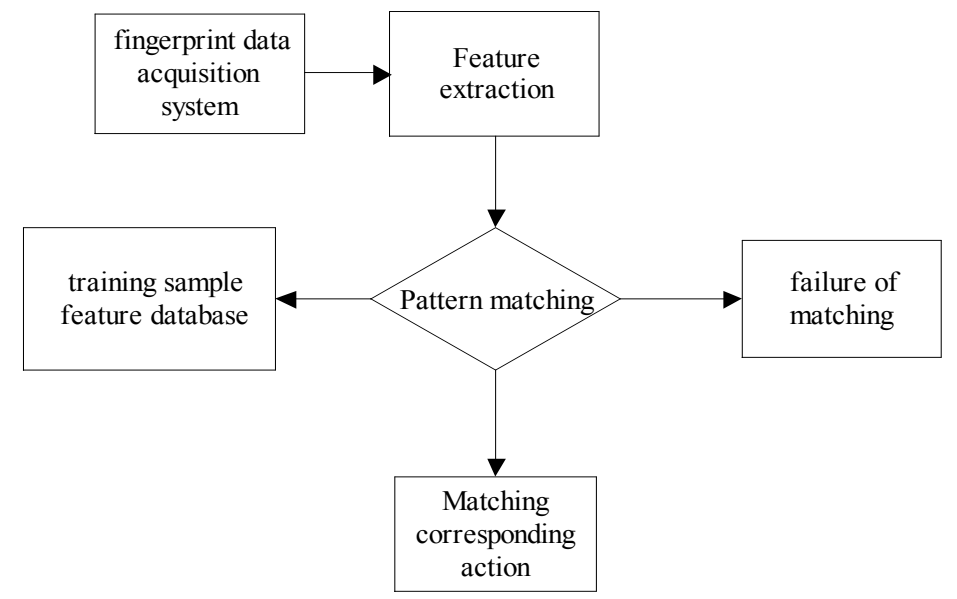

Fig. (1). Operation on biological recognition system.

feature recognition system are: capture and storage of registered biological feature sample; capture new biological feature sample and make comparison on them. The figure depicts a common biological feature recognition system, although some systems somewhat are different in the details. The main components of the recognition system are "pattern matching", and the biological feature data is collected by the sensors from the identified object, "reference database": storage of biological feature data of registered object of early period, and the "matcher" determine "reactive end" by recognition through making comparison of submitted data and reference data, and the recognition results will be released and some action based on the result of will also be developed.

Systems in early stage were realized based on principal component analysis feature extraction technique and the technical and non-parallel fed back propagation recognition technology. The problem to the method of identification of face at first followed by fingerprint identification is that it is based on a single-mode biological feature system. Singlemode biological feature systems have many problems, such as noise data, change within the class, freedom restriction, non-universality, spoofing and unacceptable error rate. This configuration-based approach is unable to make normalization of false acceptance rate, false rejection rate or disapproved rate. It proposes "artificial multi-biological feature recognition is close to biological feature recognition of face and fingerprint ", as part of its work, artificial neural networks have been completed. Feature extraction uses principal component analysis and the recognition uses feedforward back-propagation neural network. Their job is to deal with the task by identifying the face and then identifying a fingerprint. Trained neural network divide input pixel aggregation into different clusters providing results. It proposes multimode biological recognition system by use of fingerprint and facial recognition technology to identify and validate personnel. The system adopts characteristics (face and fingerprint) individual score in combination of classifier level and classification level [10-13].

Multimode biological feature systems have been proposed, which improve the accuracy and efficiency by learning, but also a framework has been determined through assessment on the performance of the system. The system does not allow a common middleware layer with a small amount of general information to handle multimode applications [14].

Document proposes a biological feature recognition system based on unique identification of an individual which uses a different image optimization technique, such as Gaussian smoothing function, lattice strong value adjustment techniques [15]. By learning different binary method, it is possible to select the draw the best results-based approach for the input thumb image. This paper also presents some image thinning algorithm. Based on the results analysis, we chose the image thinning algorithm. The problem for artificial neural network is to understand the structure of an algorithm is very difficult, since too many factors may result in excessive filtration, and the best network structure can only be determined based on experiments [6].

Document [9] introduces a method to automatically detect and use of multi-level grades fusion multimode face and fingerprint recognition system, through the replacement of the score and the true score fusion to improve the face recognition system $[16,17]$.

\section{MULTIMODE BIOLOGICAL FEATURE IDENTI- FICATION BY ADOPTION OF NEURAL NETWORKS}

This article will focus on the implementation of multimode face and fingerprint biological feature recognition by use of neural networks. Multimode biological features recognition for identity authentication has two characteristics, which are face and fingerprint systems based on principal component analysis of adaptation and multi-level sensing. A proposal for recognition system with neural network is parallel processing, while multimode biological feature system handles individual score of two approximate range of characteristics by training (face and fingerprint), and they are discontinuous interval, so that the system can efficiently generate good and accurate results. The current work is based on the ridge and Eigenface combines parallel processing, in order to achieve efficient human face and fingerprint recognition algorithm. A method proposed here is to solve the shortcomings of the previous question, and this method is effective on neural network and joint Ridge Eigenface based and face and fingerprint recognition algorithm [18]. The main purpose of this system is to reduce error rate as much 


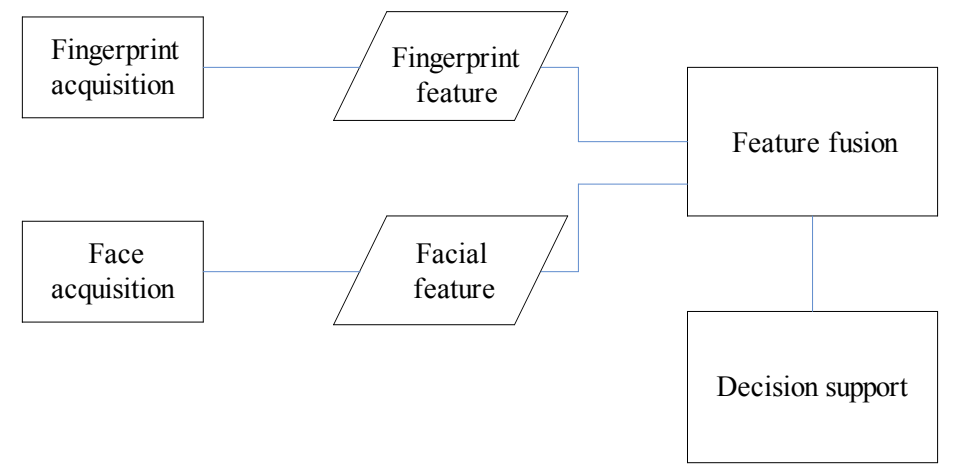

Fig. (2). Multimode biological feature recognition system by adoption of face and fingerprint characteristics.

as possible in the process of identification and authentication, and to achieve good acceptance rate by improving the performance of the system.

Implementation steps:

Acquisition phase: biological properties collected by different sensors will be collected together to generate a synthetic biological feature cluster and processed. Object or landscape images captured by camera or generated by scanning shall act as input data of the system.

Feature extraction stage: signals from different biological feature sources will first be pretreated, and the feature vector will be extracted separately through special algorithm, and then these vectors will be combined to form a composite feature vector, which offers great help to classification. The above series of implementation steps is to make better processing and interpretation for a picture in the subsequent phase [19]. These steps include noise removing, changes in the intensity of the recording, polishing, improve contrast, stretch the image texture. Another important aspect is the image storage, primarily to extract image information from a degenerate form to make it more suitable to be subsequently processed and interpreted.

Matching integration phase: in comparison with joint feature vector, when treated separately, the feature vector will produce individual match scores, and classification work may be conducted lying on each biological feature matching scores.

Estimation and decision-making stage: each mode will be subjected to pre-sorting separately at first, and multimode biological feature recognition systems will complete any integration strategy, or will put them together to improve system performance [20].

Level for system will be displayed in the figure below.

\section{EXPERIMENT}

Multimode biological recognition system database may be real or virtual. The actual multimode database is composed by biological characteristics obtained from the same individual body, while the virtual multi-mode database is consist of combination of biological features from a singlemode biological feature database and another single-mode biological feature database. Virtual multimode biological features database is based on that different biological characteristics of individual body is independent assumptions.

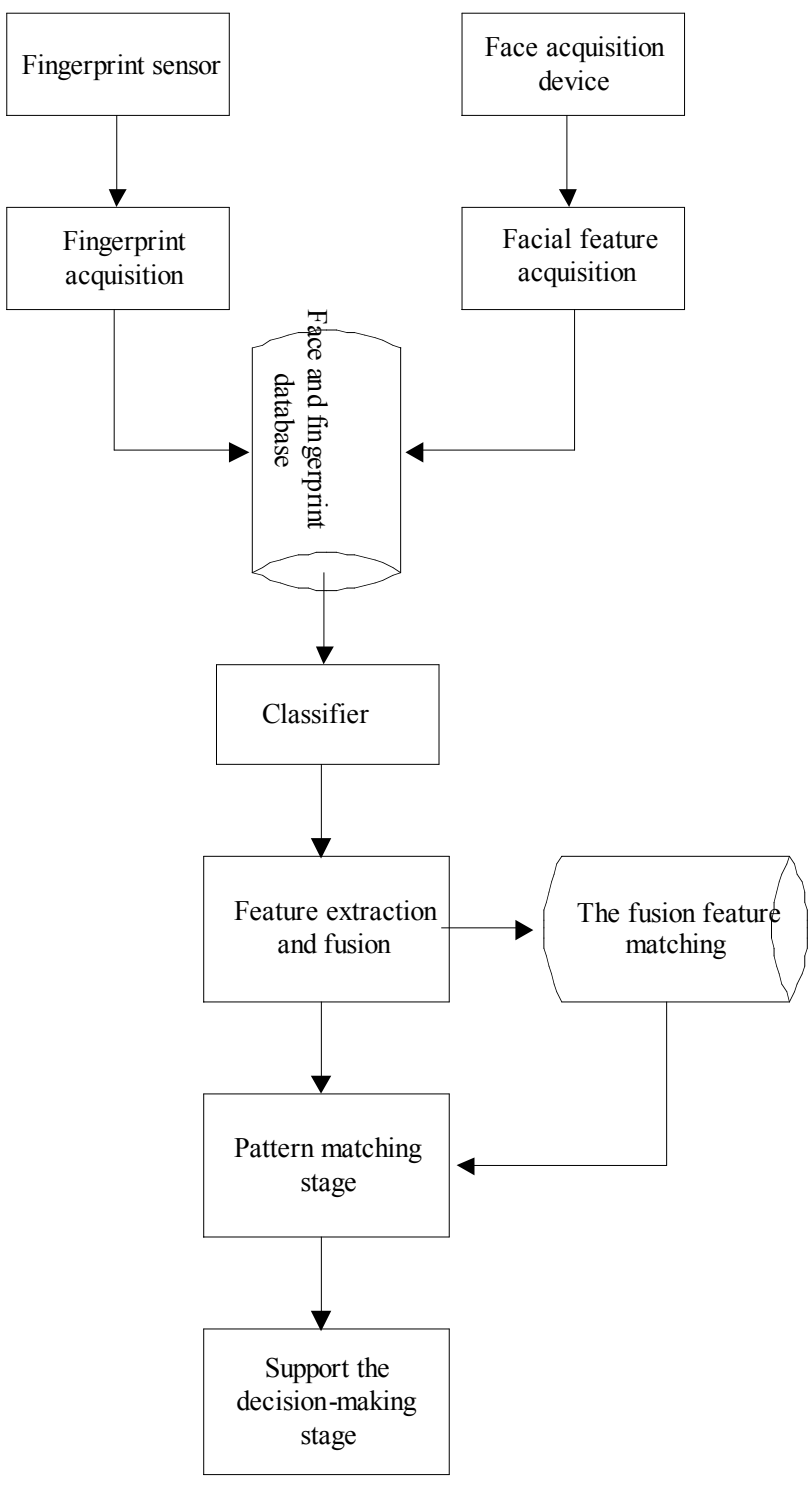

Fig. (3). Face and finger print recognition system.

Experimental data is extracted from different 200 different users aged from 20-52 year-old, including male and female, and experimental environment is in a normal computer room.

The Table 1 demonstrates the results of a single biological characteristic and of combination of the two single bio- 
Table 1. Comparison of FRR and FAR in multimode biological feature authentication system under.

\begin{tabular}{|c|c|c|c|}
\hline \multirow{2}{*}{ False Acceptance Rate (FAR) } & \multicolumn{3}{|c|}{ False Rejection Rate (FRR) } \\
\cline { 2 - 4 } & Face & Fingerprint & Multimode \\
\hline \hline $3 \%$ & $10.45 \%$ & $5.3 \%$ & $3.42 \%$ \\
\hline $0.5 \%$ & $30.40 \%$ & $6.2 \%$ & $5.12 \%$ \\
\hline $0.1 \%$ & $62.5 \%$ & $9.4 \%$ & $6.6 \%$ \\
\hline $0.045 \%$ & $76.27 \%$ & $25.2 \%$ & $15.33 \%$ \\
\hline
\end{tabular}

Table 2. Experiment results to PCA method.

\begin{tabular}{|c|c|c|c|}
\hline $\begin{array}{c}\text { Serial Number of Pictures of Face } \\
\text { and fingerprint }\end{array}$ & Successful Recognition & Non-recognition & Efficiency \\
\hline \hline 8 & 6 & 2 & $75 \%$ \\
\hline 15 & 12 & 3 & $80 \%$ \\
\hline 20 & 18 & 2 & $90 \%$ \\
\hline 25 & 21 & 4 & $84 \%$ \\
\hline 30 & 28 & 2 & $93.33 \%$ \\
\hline
\end{tabular}

Table 3. Experiment results to APCE method.

\begin{tabular}{|c|c|c|c|}
\hline $\begin{array}{c}\text { Serial Number of Pictures of Face } \\
\text { and fingerprint }\end{array}$ & Successful Recognition & Non-recognition & Efficiency (\%) \\
\hline \hline 8 & 7 & 1 & $87.5 \%$ \\
\hline 15 & 13 & 1 & $86.67 \%$ \\
\hline 20 & 19 & 2 & $95.33 \%$ \\
\hline 25 & 23 & 1 & $92 \%$ \\
\hline 30 & 29 & $96.67 \%$ \\
\hline
\end{tabular}

logical characteristics respectively. The data reveals that when a single character and integrated features of face and fingerprint have the same false acceptance rate, then the false rejection rate of single features is higher than the false rejection rate of multi-mode. The following table shows a comparison of results shown in the table. It is obvious to discover that multi-mode biological features obviously enhance the performance of the system.

As we can see from the Table 2 that the efficiency for ace and fingerprint recognition systems using principal component analysis algorithm is $93.33 \%$, and these results reflect that achievement of a better match performance results by using multimode biological feature recognition system. It is also deduced that the choice of fusion features in the multimode biological feature recognition system in operation has significant influence on the experiment results.

As we can be seen from the Table 3 that efficiency for face and fingerprint recognition systems using adaptive principal component extraction algorithm is $96.67 \%$, and the application requirements determine the fault rate, in single- mode and multimode biological feature recognition systems, it is required that there is a trade-off of the system between usability and safety.

\section{CONCLUSION}

The work done in this paper is mainly to standardization of false acceptance rate and registration failure rate and false rejection rate, and to propose reliable method to ensure the safety and integrity rate of biological feature data and to achieve a higher accuracy in comparison with single mode, while reducing identification system identifying the response time. When the high-frequency coefficients are not very sensitive to human virtual systems, multimode recognition has efficient performance in comparison with the single-mode recognition, some of the coefficients of each block have been formed, and forecasting model based on software calculation so advocated is more easily achieved on the other hand. This paper also implements a standardized combination of face and fingerprint authentication for personal biological feature system that overcomes many of the limitations of face recognition systems and fingerprint verification system. 


\section{CONFLICT OF INTEREST}

The authors confirm that this article content has no conflict of interest.

\section{ACKNOWLEDGEMENTS}

Declared none.

\section{REFERENCES}

[1] M. Zhang, Z. Lv, X. Zhang, G. Chen, and K. Zhang, "Research and Application of the 3D Virtual Community Based on WEBVR and RIA," Computer and Information Science, vol. 2, no. 1, pp. 84, 2009.

[2] T. Su, Z. Lv, S. Gao, X. Li, and H. Lv, "3D seabed: 3D modeling and visualization platform for the seabed," In: Multimedia and Expo Workshops (ICMEW), International Conference on, pp. 1-6, 2014.

[3] X. Li, Z. Lv, B. Zhang, W. Wang, S. Feng, and J. Hu, "WebVRGIS Based City Bigdata 3D Visualization and Analysis," In: Pacific Visualization Symposium (PacificVis), 2015.

[4] S. Li, Y. Geng, J. He, and K. Pahlavan, "Analysis of Threedimensional Maximum Likelihood Algorithm for Capsule Endoscopy Localization," In: 5th International Conference on Biomedical Engineering and Informatics (BMEI), Chongqing, China, pp. 721-725, 2012,

[5] Y. Geng, J. He, H. Deng and K. Pahlavan, "Modeling the Effect of Human Body on TOA Ranging for Indoor Human Tracking with Wrist Mounted Sensor," 16th International Symposium on Wireless Personal Multimedia Communications (WPMC), Atlantic City, NJ, 2013.

[6] J. Wright, A. Y. Yang, A. Ganesh, and S.S. Sastry, "Robust face recognition via sparse representation," Pattern Analysis and Machine Intelligence, IEEE Transactions on, vol. 31, no. 2, pp. 210227, 2009.

[7] Z. Zhang, J. Wang, and H. Zha, "Adaptive Manifold Learning," IEEE Transactions on Pattern Analysis and Machine Intelligence, vol. 34, no. 1, pp. 131-137, 2012.

[8] R. He, B. G. Hu, and X. T. Yuan, "Robust discriminant analysis based on nonparametric maximum entropy" Advances in Machine Learning, vol. 54, no. 3, pp. 120-134, 2009.
[9] D. Cai, X. He, and J. Han, "Spectral regression for efficient regularized subspace learning," In: Computer Vision, ICCV 11th International Conference on, pp. 1-8, 2007.

[10] M. Becker, E. Werkman, M. Anastasopoulos, and T. Kleinberger, "Approaching ambient intelligent home care systems," In: Pervasive Health Conference and Workshops, pp. 1-10, 2006.

[11] M. Floeck, and L. Litz, "Integration of Home Automation Technology into an Assisted Living Concept," Assisted Living SystemsModels, Architectures and Engineering Approaches, 2007.

[12] J. Fraile, J. Bajo, and J. Corchado, "Amade: Developing a multiagent architecture for home care environments," In: 7th IberoAmerican Workshop in Multi-Agent Systems, 2008.

[13] T. Alex, B. Laurent, M. Piuzzi, Z. Lu, M. Chavent, M. Baaden, O. Delalande, P. Bourdot, and N. Ferey, "Advances in Human-Protein Interaction-Interactive and Immersive Molecular Simulations," InTech, 2012.

[14] Z. Lv, A. Halawani, S. Feng, S. ur Rehman, and H. Li, "Touch-less Interactive Augmented Reality Game on Vision Based Wearable Device," Personal and Ubiquitous Computing, 2015.

[15] S. Dang, I. Ju, D. Matthews, and X. Feng "Efficient solar power heating system based on lenticular condensation," In: International Conference on Information Science, Electronics and Electrical Engineering (ISEEE), pp. 26-28, 2014.

[16] Z. Lv, L. Feng, S. Feng, and H. Li, "Extending Touch-less Interaction on Vision Based Wearable Device," IEEE Virtual Reality (VR). IEEE, 2015.

[17] Y. Geng, J. He, H. Deng and K. Pahlavan, "Modeling the Effect of Human Body on TOA Ranging for Indoor Human Tracking with Wrist Mounted Sensor," 16th International Symposium on Wireless Personal Multimedia Communications (WPMC), Atlantic City, NJ, 2013.

[18] Y. Geng, J. He, and K. Pahlavan, "Modeling the Effect of Human Body on TOA Based Indoor Human Tracking," International Journal of Wireless Information Networks, vol. 20, no. 4, pp. 306-317, 2013.

[19] Y. Geng, and K. Pahlavan, "On the Accuracy of RF and Image Processing Based Hybrid Localization for Wireless Capsule Endoscopy," IEEE Wireless Communications and Networking Conference (WCNC), 2015.

[20] J. He, Y. Geng, and K. Pahlavan, "Toward Accurate Human Tracking: Modelling Time-of-Arrival for Wireless Wearable Sensors in Multipath Environment," IEEE Sensor Journal, vol. vol. 14, no. 11, pp. 3996-4006, 2014.

(c) Xiaosong and Zhiqing; Licensee Bentham Open.

This is an open access article licensed under the terms of the (https://creativecommons.org/licenses/by/4.0/legalcode), which permits unrestricted, noncommercial use, distribution and reproduction in any medium, provided the work is properly cited. 